\title{
Systemic oxidative stress and antioxidant capacity in cancer patients
}

\author{
Leimkuhler $\mathrm{M}^{1}$, Bourgonje $\mathrm{AR}^{2}$, van Goor $\mathrm{H}^{3}$, van Leeuwen $\mathrm{BL}^{1}$ and Bock de $\mathrm{GH}^{4 *}$ \\ ${ }^{1}$ Department of Surgical Oncology, University Medical Center Groningen, University of Groningen, Groningen, The Netherlands \\ ${ }^{2}$ Department of Gastroenterology and Hepatology, University Medical Center Groningen, University of Groningen, Groningen, Hanzeplein 1, 9713 GZ Groningen, \\ The Netherlands \\ ${ }^{3}$ Department of Pathology and Medical Biology, University Medical Center Groningen, University of Groningen, Groningen, Hanzeplein 1, 9713 GZ Groningen, \\ The Netherlands \\ ${ }^{4}$ Department of Epidemiology, University Medical Center Groningen, University of Groningen, Groningen Hanzeplein 1, 9713 GZ Groningen, The Netherlands
}

\begin{abstract}
Various factors impact the outcome of patients with the diagnosis of cancer. Common treatment modalities of cancer, including surgery, radiation therapy and cytostatic agents, all lead to a systemic inflammatory reaction. In particular, this reaction is of physiological importance as it is crucial for patient recovery. However, in some patients, a self-perpetuating inflammatory response develops due to the presence of unfavorable risk factors, several of which are still unknown, but might lead to a worse disease prognosis. Inflammation has been intimately associated with oxidative stress, that is characterized by an imbalance between pro-oxidants, also termed reactive species, and antioxidants. Systemically, oxidative stress can be quantified by measuring thiols (R-SH, sulfhydryl compounds), which are considered to be regulatory nodes within the extracellular antioxidant network. Most importantly, thiol measurements in serum or plasma form a robust and powerful read-out of the in vivo reduction-oxidation (redox) status as thiols are highly redox-active and thus readily oxidized by circulating reactive species. Therefore, systemic quantification of thiols might be a valuable addition to the clinically available diagnostic and prognostic armamentarium as it is able to reliably capture the overall balance between oxidants and the antioxidant capacity of patients. In this review, we summarized the currently available literature on thiol levels as amendable markers for oxidative stress in patients with lung, prostate and colorectal cancer. Total thiols, native (free) thiols and disulfide levels are significantly altered in these patients compared to healthy individuals. In general, these findings indicate that the extracellular antioxidant capacity is severely affected in patients with these types of cancer. Moreover, lower thiol levels are associated with a lowered overall survival. Future research should focus on exploration of the clinical significance of thiols in cancer.
\end{abstract}

\section{Introduction}

Over the past decades, the outcome of various cancer treatments has improved in terms of overall and disease-free survival. However, treatment is often intensive and might lead to (severe) complications and a diminished quality of life for the patient. Though essential for patient recovery, an exaggerated systemic inflammatory response is associated with the occurrence of complications after cancer treatment $[1,2]$. Systemic inflammation is intimately associated with oxidative stress [1]. Oxidative stress is characterized by an excessive production of reactive species and decreased availability of antioxidants, also referred to as a redox imbalance [3-6]. A redox imbalance becomes apparent when the antioxidant capacity of the patient is decreased. [1,2,5,7]. Increased levels of oxidative stress have been suggested to be related to oncogenic stimulation, such as uncontrolled cell proliferation and DNA or protein modification $[3,5,7,8]$.

Thiols (R-SH, sulfhydryl compounds) are central to the extracellular non-enzymatic antioxidant capacity, and can be easily measured in patients' serum or plasma using established colorimetric detection methods $[9,10]$. Generally, thiols are considered as a robust and powerful read-out of an individual's systemic reductionoxidation (redox) status as they are rapidly oxidized by nearby reactive species $[5,7,11,12]$. Thus, systemic oxidative stress is associated with reduced levels of thiols, whereas higher levels of thiols are considered representative of a more favorable redox status in vivo [13]. Thiols are characterized by sulfhydryl groups (-SH), which are responsible for their potent antioxidant activity, and can be oxidized by reactive species to form reversible disulfide compounds (RSSR'), a dynamic equilibrium which is commonly termed thiol-disulfide homeostasis, as illustrated in Figure 1 [7,14-16]. The plasma thiol pool mainly consists of albumin and protein thiols, while the remainder is composed by low-molecularweight (LMW) thiols (e.g. cysteine or glutathione) [11]. Thiol-disulfide homeostasis is a dynamic physiological entity representing the net result of kinetically controlled intra- and extracellular exchange reactions and has important functions in different cellular processes, such as proliferation, inflammation and apoptosis [3,8,11,15,17]. Systemic oxidative stress is reflected by decreased levels of reduced (free) thiols (native thiols) and total thiols, and increased levels of disulfides [18]. A reduced antioxidant capacity has been demonstrated in various types of cancers [19]. Measuring the antioxidant capacity, therefore, can provide us with valuable information about the redox status of cancer patients and their buffering capability to counterbalance oxidative stress. To know the response of thiol levels to various treatments and their clinical

${ }^{\star}$ Correspondence to: G.H. de Bock, University Medical Center Groningen, University of Groningen, Groningen Hanzeplein 1, 9713 GZ Groningen, The Netherlands, E-mail: g.h.de.bock@umcg.nl

Received: January 07, 2020; Accepted: January 21, 2020; Published: January 24 2020 
implications might help to improve risk stratification of cancer patients. In this review, we summarize the currently available literature on thiol redox status specifically in patients with lung, prostate and colorectal cancer. In addition, we describe how systemic thiol levels are related to patient outcomes. For that, in collaboration with an experienced medical research librarian, a search strategy was developed and a search was performed in PubMed (Supplementary 1, date of search: 16/01/2020). Available literature was screened on title and in case of potential relevance, the abstract was screened. Ten publications were included in this review. Thiol redox status in lung, prostate and colorectal cancer

\section{Lung cancer}

Three studies described the thiol redox status in patients affected by lung cancer (Table 1). All of these studies found total thiol levels, and two studies found native thiol levels and disulfide levels to be reduced in lung cancer patients compared to healthy controls $[5,19,20]$. These results indicate that the extracellular antioxidant capacity is generally compromised in these patients. In addition, one study described that total thiol levels were associated with ageing and a history of smoking

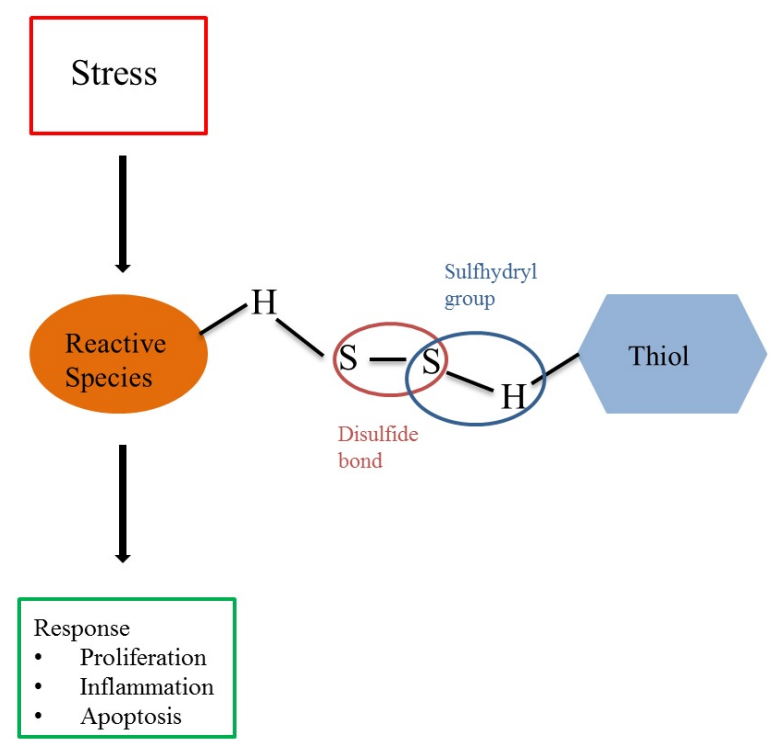

Figure 1. Thiol-disulfide homeostasis
[19], whereas another study described that current smoking, COPD, the histological subtypes of the tumor or arterial hypertension were not associated with thiol/disulfide homeostasis in this patient group [20], although this might be the result of a lack of statistical power. Patients with lower total thiol levels showed a significantly lower Karnofsky performance score [5]. In addition, patients with lower native thiol levels or disulfide levels showed a decreased overall survival $[5,20]$.

\section{Prostate cancer}

Five studies reported on the thiol redox status in patients with prostate cancer $[8,17,19,21,22]$. All, but one [21], described significantly lower levels of total thiols in patients with prostate cancer compared to healthy controls, and three described lower native thiol levels $[8,17,22]$, but only two studies also described lower disulfide levels $[8,22]$. Factors that were associated with lower thiol levels in prostate cancer constituted older age, lower educational level, current or previous smoking and a higher BMI [19]. Interestingly, in one study, it was demonstrated that native and total thiol levels were increased six months after prostatectomy [8], suggesting that higher thiol levels are influenced by tumor presence.

\section{Colorectal cancer}

In patients with colorectal cancer, total thiol levels were lowered compared to healthy subjects [19], as well as the disulfide level [14]. In patients with more aggressive tumors, the disulfide level was even lower than in patients with slow growing tumors [14]. In addition, lower thiol levels were associated with a higher age and a higher BMI [19].

\section{Conclusion and general discussion}

In general, the extracellular non-enzymatic antioxidant capacity, as reflected by the level of thiols, is significantly compromised in patients with lung cancer, prostate cancer or colorectal cancer. The same phenomenon has been described for other tumor types as well $[14,19,23,24]$. Lower thiol levels have also been associated with a reduced overall survival in patients with oral squamous carcinoma [24]. Similar results were seen in patients with multiple myeloma, where treated patients presented with lower thiol levels than patients who were about to initiate treatment [25]. In addition, lower thiol levels might have a prognostic value in the development of chemotherapyinduced cardiac toxicity [15]. It has previously been observed that total thiol levels, native thiol levels and disulfide levels are decreased after a

Table 1. Available data on thiol and disulfide levels in cancer patients and healthy controls ( ${ }^{\times}$median (range); na=not available; *measurements out of graph)

\begin{tabular}{|c|c|c|c|c|c|c|c|}
\hline Reference & Number of participants & $\begin{array}{c}\text { Total thiol } \\
\text { Patients } \\
\text { Mean (SD) } \mu \mathrm{mol} / \mathrm{L}\end{array}$ & $\begin{array}{c}\text { Total thiol } \\
\text { Healthy Controls } \\
\text { Mean (SD) } \mu \mathrm{mol} / \mathrm{L}\end{array}$ & $\begin{array}{l}\text { Native thiol } \\
\text { Patients } \\
\text { Mean (SD) } \\
\mu \mathrm{mol} / \mathrm{L}\end{array}$ & $\begin{array}{c}\text { Native thiol } \\
\text { Healthy Controls } \\
\text { Mean (SD) } \mu \mathrm{mol} / \mathrm{L}\end{array}$ & $\begin{array}{c}\text { Disulfide } \\
\text { Patients } \\
\text { Mean (SD) } \mu \mathrm{mol} / \mathrm{L}\end{array}$ & $\begin{array}{c}\text { Disulfide } \\
\text { Healthy Controls } \\
\text { Mean (SD) } \mu \mathrm{mol} / \mathrm{L}\end{array}$ \\
\hline \multicolumn{8}{|c|}{ Lung cancer } \\
\hline Dirican (5) & $\begin{array}{c}35 \text { patients, } 35 \text { healthy } \\
\text { controls }\end{array}$ & $300.6(68.9)$ & $354.0(79.5)$ & $266.1(64.5)$ & $313(78.2)$ & $17.2(5.6)$ & $20.2(4.4)$ \\
\hline Karatas (20) & $\begin{array}{c}60 \text { patients, } 60 \text { healthy } \\
\text { controls }\end{array}$ & $256.7(69.1)$ & $314.7(49.4)$ & $217.1(6.73)$ & $273.9(49.1)$ & $18.2(9.9-38.9)^{\times}$ & $19.8(17.5-32.7)^{\times}$ \\
\hline \multicolumn{8}{|c|}{ Prostate cancer } \\
\hline Sönmez (17) & $\begin{array}{c}29 \text { patients, } \\
30 \text { healthy controls }\end{array}$ & $345.1(56.7)$ & $463.0(35.8)$ & $303.5(48.5)$ & $424.0(35.6)$ & $19.6(7.19)$ & $19.8(9.8)$ \\
\hline Hanikoglu (8) & $\begin{array}{c}18 \text { patients } \\
17 \text { healthy controls }\end{array}$ & $378.8(46.5)$ & $462.3(61.5)$ & $350.7(46.35)$ & $419.8(54.9)$ & $14.0(10.5)$ & $21.3(6.4)$ \\
\hline Tokgöz (26) & 22 patients & $470.2(47.5)$ & na & $441.5(44.9)$ & na & $14.3(2.7)$ & na \\
\hline Solakhan (22) & $\begin{array}{c}25 \text { patients, } \\
25 \text { healthy controls }\end{array}$ & $150.3(45.3)$ & $191.0(32.3)$ & $118.4(36.8)$ & $144.1(21.2)$ & $15.9(7.0)$ & $23.4(10.1)$ \\
\hline Battisti (21) & $\begin{array}{c}55 \text { patients, } \\
55 \text { healthy controls }\end{array}$ & $145.0 *$ & $180.0^{*}$ & na & na & na & na \\
\hline
\end{tabular}


prostate biopsy [26], but it is unknown whether this has any prognostic significance. These results might lead to the assumption that patients should be treated with antioxidants to improve their prognosis. In the past, researchers focused on antioxidant therapy to improve disease prognosis, however, results from these studies were disappointingly unsuccessful. For example, it was found that long-term supplementation with antioxidants can even lead to a promotion of metastasis, as shown in a mouse model of vitamin E effects on lung cancer metastasis [27]. This might be the consequence of the complexity of the redox system, which in general serves a physiological purpose and is only harmful in the situation of overproduction of reactive species. Therefore, targeted therapeutic modulation, taking into account patient comorbidity and varying phenotypes of each patient, might be crucial in order to achieve successful therapy.

Measurement of serum or plasma thiols and the determination of thiol/disulfide homeostasis may be a valuable strategy to determine the systemic redox status of an individual patient [28]. However, thiol levels can be influenced by a multitude of factors and are not diseasespecific. In addition, the studies discussed in this review have been performed in highly selected patients and control groups, without various comorbidities that influence systemic thiol levels $[8,22]$. This might overestimate the influence of thiols on the prognosis, as results are not generalizable to the general population of patients. In the future, prospective, longitudinal studies are required, that focus on the response of thiol-based redox signaling to various treatments and its clinical implications. Furthermore, studies should focus on further unraveling the redox system and its association with individual characteristics, such as genes, proteins or the gut microbiome, to disclose major determinants of the redox system.

\section{References}

1. Murata M (2018) Inflammation and cancer. Environ Health Prev Med 23: 50-51.

2. Plas M, de Haan JJ, van der Wal-Huisman H, Rutgers A, Absalom AR, et al. (2019) The systemic impact of a surgical procedure in older oncological patients. Eur J Surg Oncol 45: 1403-1409.

3. Grek CL, Tew KD (2010) Redox metabolism and malignancy. Curr Opin Pharmacol 10: $362-368$.

4. Ozler S, Oztas E, Tokmak A, Ergin M, Isci E, et al. (2016) The association of thiol/ disulphide homeostasis and lipid accumulation index with cardiovascular risk factors in overweight adolescents with polycystic ovary syndrome. Clin Endocrinol (Oxf) 84: 516-523.

5. Dirican N, Dirican A, Sen O, Aynali A, Atalay S, et al. (2016) Thiol/disulfide homeostasis: A prognostic biomarker for patients with advanced non-small cell lung cancer? Redox Rep 21: 197-203.

6. Elnakish MT, Hassanain HH, Janssen PM, Angelos MG, Khan M (2013) Emerging role of oxidative stress in metabolic syndrome and cardiovascular diseases: important role of Rac/NADPH oxidase. J Pathol 231: 290-300.

7. Eroglu S, Haskul I, Aziz V, Yurtcu E, Karatas F, et al. (2017) Dynamic thiol/disulphide homeostasis in patients with Uterine Myoma. Eur J Obstet Gynecol Reprod Biol 216: 24-26.

8. Hanikoglu F, Hanikoglu A, Kucuksayan E, Alisik M, Gocener AA, et al. (2016) Dynamic thiol/disulphide homeostasis before and after radical prostatectomy in patients with prostate cancer. Free Radic Res 50(sup1): S79-S84.
9. Ellman gl (1959) Tissue sulfhydryl groups. Arch Biochem Biophys 82: 70-77.

10. Hu ML, Louie S, Cross CE, Motchnik P, Halliwell B (1993) Antioxidant protection against hypochlorous acid in human plasma. J Lab Clin Med 121: 257-262.

11. Guney T, Kanat IF, Alkan A, Alisik M, Akinci S, et al. (2017) Assessment of serum thiol/disulfide homeostasis in multiple myeloma patients by a new method. Redox Rep 22: $246-251$.

12. Yuan K, Liu Y, Chen HN, Zhang L, Lan J, Gao W, et al. (2015) Thiol-based redox proteomics in cancer research. Proteomics 15: 287-299.

13. Banne AF, Amiri A, Pero RW (2003) Reduced level of serum thiols in patients with a diagnosis of active disease. J Anti Aging Med 6: 327-334.

14. Erel O, Neselioglu S (2014) A novel and automated assay for thiol/disulphide homeostasis. Clin Biochem 47: 326-332.

15. Topuz M, Sen O, Kaplan M, Akkus O, Erel O, et al. (2017) The role of thiol/disulphide homeostasis in anthracycline associated cardiac toxicity. Int Heart J 58: 69-72.

16. Cortese-Krott MM, Koning A, Kuhnle GGC, Nagy P, Bianco CL, et al. (2017) The reactive species interactome: Evolutionary emergence, biological significance, and opportunities for redox metabolomics and personalized medicine. Antioxid Redox Signal 27: 684-712.

17. Sonmez MG, Kozanhan B, Deniz CD, Gog er YE, Kilinc MT, et al. (2018) Is oxidative stress measured by thiol/disulphide homeostasis status associated with prostate adenocarcinoma? Cent Eur J Immunol 43: 174-179.

18. Kundi H, Ates I, Kiziltunc E, Cetin M, Cicekcioglu H, et al. (2015) A novel oxidative stress marker in acute myocardial infarction; thiol/disulphide homeostasis. Am J Emerg Med 33: 1567-1571.

19. Gao X, Wilsgaard T, Jansen E, Xuan Y, Anusruti A, et al. (2020) Serum total thiol levels and the risk of lung, colorectal, breast and prostate cancer: A prospective case-cohort study. Int J Cancer 146: 1261-1267.

20. Karatas F, Acat M, Sahin S, Inci F, Karatas G, et al. (2019) The prognostic and predictive significance of serum thiols and disulfide levels in advanced non-small cell lung cancer. Aging Male 16: 1-10.

21. Battisti V, Maders LD, Bagatini MD, Reetz LG, Chiesa J, et al. (2011) Oxidative stress and antioxidant status in prostate cancer patients: relation to Gleason score, treatment and bone metastasis. Biomed Pharmacother 65: 516-524.

22. Solakhan M, Cicek H, Orhan N, Yildirim M (2019) Role of native Thiol, total Thiol and dynamic Disulphide in diagnosis of patient with prostate cancer and prostatitis. Int Braz J Urol 45: 495-502.

23. Inal BB, Emre HO, Baran O, Ahmedov M, Ozdemir AF, et al. (2017) Dynamic thioldisulphide homeostasis in low-grade gliomas: Preliminary results in serum. Clin Neurol Neurosurg 161: 17-21.

24. Patel BP, Rawal UM, Dave TK, Rawal RM, Shukla SN, et al. (2007) Lipid peroxidation, total antioxidant status, and total thiol levels predict overall survival in patients with oral squamous cell carcinoma. Integr Cancer Ther 6: 365-372.

25. Ellidag HY, Eren E, Aydin O, Yildirim M, Sezer C, et al. (2014) Multiple myeloma: relationship to antioxidant esterases. Med Princ Pract 23: 18-23.

26. Tokgoz H, Tas S, Giray O, Yalcinkaya S, Tokgoz O, et al. (2017) The change in serum Thiol/Disulphide homeostasis after transrectal ultrasound guided prostate biopsy. Int Braz J Urol 43: 455-461.

27. Wiel C, Le Gal K, Ibrahim MX, Jahangir CA, Kashif M, et al. (2019) BACH1 Stabilization by Antioxidants Stimulates Lung Cancer Metastasis. Cell 178: 330-345. e22.

28. Ozyazici S, Karateke F, Turan U, Kuvvetli A, Kilavuz H, et al. (2016) A Novel oxidative stress mediator in acute appendicitis: Thiol/disulphide homeostasis. Mediators Inflamm 6761050 .

Copyright: $@ 2020$ Leimkuhler M. This is an open-access article distributed under the terms of the Creative Commons Attribution License, which permits unrestricted use, distribution, and reproduction in any medium, provided the original author and source are credited. 